\title{
IoT Based Water Consumption and Quality Monitoring System
}

\author{
P.Selvaraj, S. Prabakaran, Sayani Chatterjee, Ambika Srivastava
}

\begin{abstract}
Today, advancement in technology and increase in population has led to over-consumption of water by households. Also, because of elevated pollution levels, water gets contaminated and can be unfit for usage. These days, a lot of water conservation and quality checking systems use Internet of Things (IoT). However, because of shortcomings of the equipment, IoT independent from anyone else can't process, store and assure the nature of these administrations. Thus, to solve this problem, IoT can be combined with two infrastructures to enhance the quality and accessibility of these administrations, cloud and fog computing. This research paper proposes a model for monitoring the water quality parameters using sensors and keeping a check on water consumption through a pricing system. This proposed model uses IoT for acquisition of data, fog layer for short-term storage and data pre-processing, and cloud layer for data analysis, processing and long-term storage.
\end{abstract}

Index Terms: Cloud Computing, Fog Computing, Internet of Things, Water Quality Index.

\section{INTRODUCTION}

Water is a huge asset for the survival of human beings. Today, due to constrained water resources and expanding populace water has turned into a fundamental asset. Clean and safe drinking water is the most vital resource for humankind. As contaminated water is one of the leading causes for most of the diseases nowadays there is a requirement for real-time water quality checking framework. World Economic Forum positioned drinking water crisis as one of the worldwide hazard, because of which around 200 youngsters are dying every day. Drinking contaminated water alone causes around 3.4 million deaths for every year. In spite of the headways in innovation, adequate quality measures are absent to gauge the nature of drinking water. The strategies utilized for water quality evaluation at present include the accumulation of random samples of water at different areas week after week or month to month and examining them in the research laboratories [1]. This methodology isn't much effective on the grounds that they have certain disadvantages,

Revised Manuscript Received on July 05, 2019.

P.Selvaraj, Department of IT, SRM IST, Kattankulathur, Chennai, India.

S. Prabakaran, Department of CSE, SRM IST, Kattankulathur, Chennai, India.

Sayani Chatterjee, Ambika Srivastava Department of IT, SRM IST, Kattankulathur, Chennai, India. for example, long-time consumption, water tests from only a couple of regions can be resolved at the same time. This technique additionally includes manual work to screen the nature of water consistently.

In order to meet the requirements of agriculture, businesses and the household division, it is essential that enough water supply is available. Every day the demand for water supply is increasing at an alarming rate. But unfortunately improper water management, scarcity of water and inadequate consumption measures have made a hard-to-oversee situation. Manual labor is used to control the appropriation of water from one region to another. The water consumption by households is not monitored appropriately which leads to wastage. Every day the manner in which the world interacts changes. A large number of applications and systems have seen the implementation of cloud computing in the last couple of years. The cloud, however, is not capable of alone handling simply technical needs and essentially fundamental applications [2]; the Internet of things (IoT) has wound up being the latest innovative pattern with respect to cloud computing [3]. In water conservation and quality checking applications, wireless sensor systems (WSN) have begun assuming an enormous job in the manner in which water supply is being observed [4][5]. Wireless sensors as gadgets are connected to the water supply channels and consumer's tank in order to achieve analytical goals. According to the requirement, the sensors can be of different sizes.

WSN's produces tremendous amount of raw data which reduces the efficiency of the system. The system must be context-sensitive so that it can reject redundant data. The data accumulated by the sensors located in the water pipelines will be forwarded to the fog nodes [6]. The fog nodes perform the task of job aggregation, analysis and also provides short-term storage. Henceforth, IoT gives an elective methodology in which the smart gadgets are used in a common architecture. The data from the sensors can then be moved to a cloud server. It is not practical to have typical sensor-cloud architecture for many water conservation and quality monitoring systems. Also, there is always a chance of the data being at risk because of data center failure or network failure. In such cases, fog computing can play an important role [7][8][9]. If only cloud is used, then it may result in delay while trading of data. Subsequently, the exchange of such huge measures of

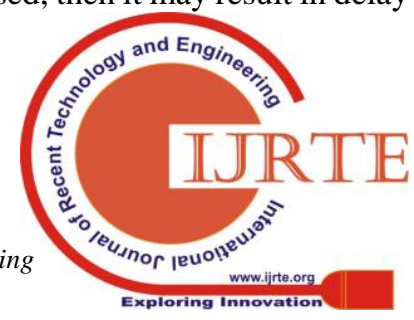


information forward and backward is not a proficient option because of latency issues as well as because of security. Hence, the cloud computing framework must be of a distributed type. This framework is known as edge computing. The edge devices are incorporated with the cloud resources through a fog computing layer and thus expanding the current cloud infrastructure.

In this paper, we endeavor to enhance the existing water consumption and quality monitoring services [10] by executing the concept of fog computing. The application resides in the cloud as well as in the gadgets and sensors closest to the consumer units. The principle objective of our proposed work is to give consistent record of the quality [11] and consumption of water. Also, the issues like cost, maintenance etc. are solved because of the distributed nature of data handling and storage in cloud data centers. We talk about the different computational tasks associated with our model that will be performed in the cloud, devices and sensors or in the fog layer.

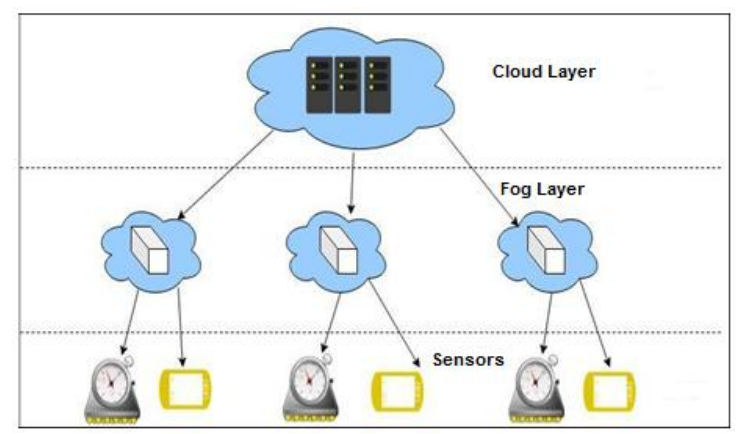

Figure 1. The tiers of IoT

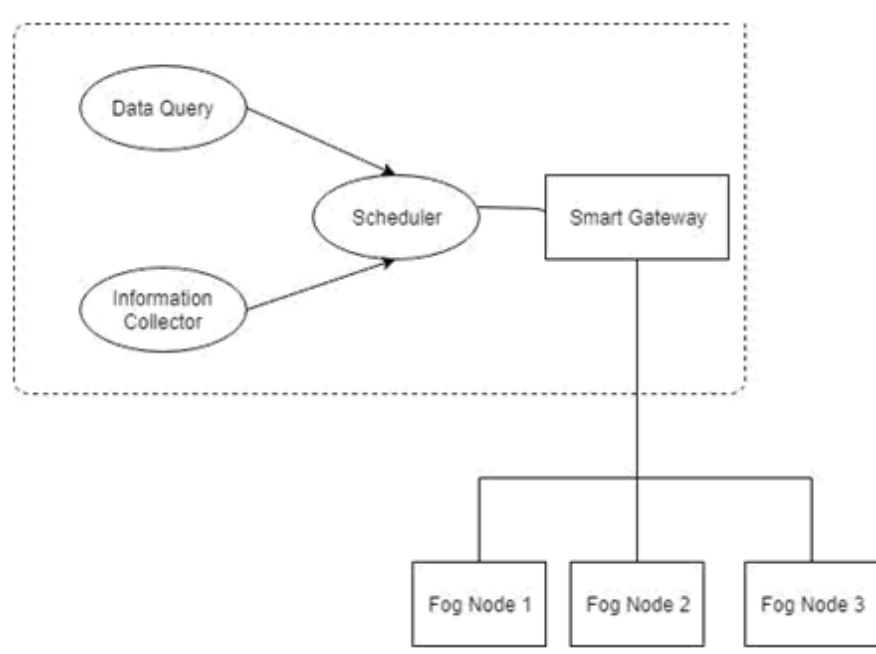

Figure 2. Fog Layer's Framework

The data exposure is constrained because it doesn't need to head back and forth in the system. This improves the security of data passing in the system. The services that the cloud performs can be conveyed to different layers so that the overhead time taken because of to and fro movement of information between the cloud and the devices is diminished. Fig. 1 and Fig. 2 depict the design of a basic fog layer with respect to the sensors and the cloud. In the second section of the paper, we examine the expectations, fog computing, and different difficulties that we have to face in this process of execution of fog along with cloud computing. The next section talks about the architecture of the system and its respective functionalities. The results are discussed in the succeeding section and analysis is done. The conclusion is given in the final section.

\section{LITERATURE REVIEW}

Fog computing is a distributed decentralized computing infrastructure in which data, compute, storage and applications are located between the data source and the cloud. The basic idea of fog computing is to bring the analytic tasks of data centers into fog nodes which are stationed before the boundary of the cloud system. We allude to these fog nodes as the fog layer. Fog computing brings the advantages and power of the cloud closer to where data is created and acted upon available information [12]. A lot of application fields can benefit from fog computing. These applications have to process a large amount of data. The following listed are the application fields that can benefit from fog along with cloud computing.

Bonomi F, Milito R, Natarajan P \& Zhu J., 2014 presented fog Computing as hierarchical and distributed platform service for processing, short-term storage. It also actualized that the usage of fog computing along with IoT in various utility services can decrease the latency and make the system more efficient. Dastjer diet la., 2016 reported that IoT generates massive amount of data that becomes difficult to handle using traditional systems, even with cloud computing although it enables innovations. The paper described the potentials and advantages of Internet of Things clubbed with fog computing like utility services, gaming, AR etc [13].

Zao JK et al., 2014 noted that for augmented reality (AR), overlaying valuable data onto the physical world progressively is vital. The paper describes the system architecture and also the technologies involved like Machine Learning, Data Web, Publisher-Subscriber Protocol and Fog/Cloud Computing. Utilizing fog computing will help accomplish this part of augmented reality more efficiently. Chen et al., 2010 reported the problems faced by using the traditional health systems due to delay in processing and sending notification. This paper presented a model of a smart gateway for the utilization of WSN in the health monitoring systems .The system uses multi-tier fog computing architecture which eases the movement of data from the body sensors to the remote data centers. The system transmits reports at real time in a low power embedded system [14].

Hart WE \& Murray R., 2010 presented a Contamination Warning System (CWS) that is a efficient approach for the detection of water contaminants from the water distribution channels. It uses an optimized strategy for the placement of the sensors. This optimized mechanism helps in rapid detection of 
pollutants. Petri I, Yuce B, Kwan A Rezgui Y., 2018 presented an analytics system that uses real time data enabling more informed and dynamic approach for managing water resources. The paper presented a system that uses Artificial Intelligence techniques to provide real-time support for managing the water resources and thereby protecting biodiversity. This system uses neural network engine for predicting river level based on dependency. Hong et al., 2013 presented Mobile Fog, which is a programming model for Internet applications that are geographically distributed and latency-sensitive. The paper tests various use cases for the model with network of camera and applications connected to various vehicles which helps in displaying the efficiency of the Mobile Fog. And also performance is measured by using simulation toolkits [15].

Pham XQ \& Huh EN., 2016 presented a task-scheduling algorithm for distribution of tasks between the fog and cloud layer. This distribution of tasks helps in reducing the workload on the cloud layer and thereby reduces the latency and improves efficiency of the system. The paper also presented the advantages of this scheduling algorithm over other existing algorithms. The main objective of this research paper is to enhance water quality analysis and conservation systems that are built on IoT so that the data is stored and processed efficiently [16]. For this, fog layer can be implemented that reduces the latency of water conservation and quality analysis systems and also sanctions real-time monitoring. Also, pricing metrics can be utilized to record water usage.

\section{IOT BASED WATER CONSUMPTION AND QUALITY MONITORING SYSTEM ARCHITECTURE}

In this portion of the paper, we propose a water consumption and quality monitoring system which has a tri-layered architecture. This system utilizes the cloud computing facility clubbed with fog computing. The three levels of infrastructure involves the sensor layer which forms the base layer, the second layer consists of the fog nodes which helps in pre-processing computation and the top layer is the cloud layer which is responsible for all the necessary actions to be taken according to the scenario.

The raw data collected by the sensors from the water pipelines flows across the tri-layered infrastructure. The low-level layer of the architecture consists of the various water quality parameter sensors i.e. $\mathrm{pH}$ sensor, turbidity sensor, conductivity sensor etc. These sensors are fitted in the water supply pipelines which facilitates the sensing of accurate data[17][18]. The data sensed by these sensors in this layer is forwarded to the second layer of operation.

The data received by the fog nodes from the base layer undergoes several layers of pre-processing computation. This layer reduces the workload of the cloud layer by distributing the various jobs amongst themselves. After segregating the various tasks, the pre-processed data is sent to the cloud layer for the necessary actions to be undertaken. Fig. 3 demonstrates the architecture of the system and Fig. 4 depicts the flow of working of the system.

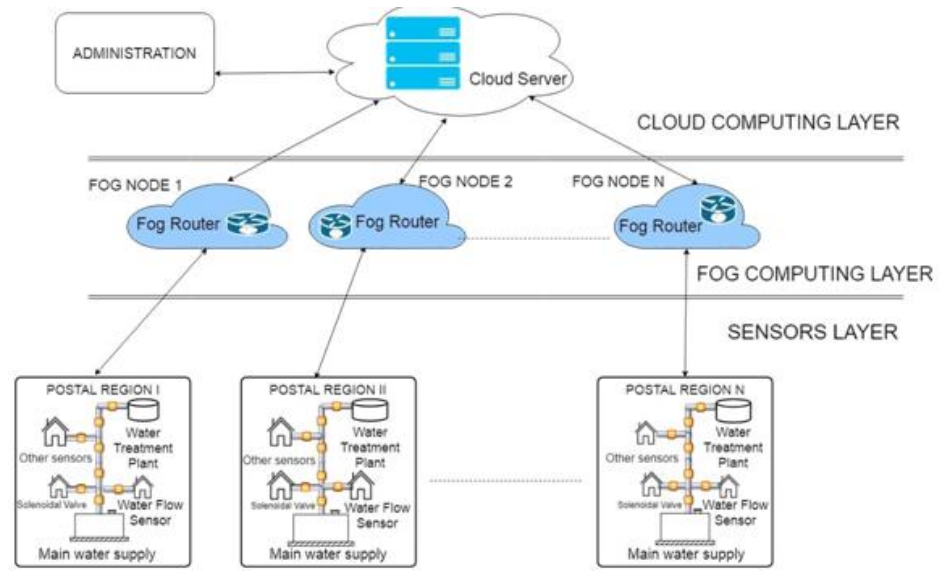

Figure 3. Architecture of the proposed system

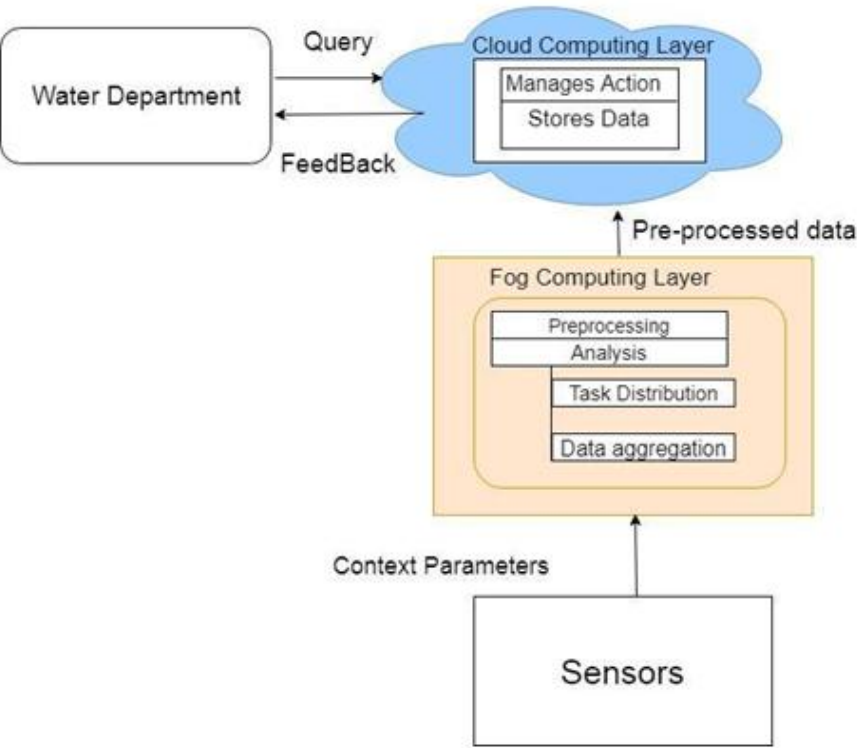

Figure 4. Schematic outline of the proposed system.

\section{A. Functionalities}

This section explains the different functionalities of the system according to the different layers of the architecture.

\section{B. Data Sensing}

In this layer, various water quality parameter sensors are placed in the water supply pipelines[19][20][21]. When the water flows through the pipelines, the sensor sense the quality parameters and forwards the raw data to the next layer of computation. The water flow sensors placed in the consumer's tank senses the consumption of water by individual household and directs it to the fog nodes for further computation. In our implementation the sensors are connected to the Node MCU board for sensing the data and sending it to the next layer. The following Figure 4 depicts the sensors connected to the Node MCU board.

\section{Pre-processing and Analytical Operations}


The fog layer which consists of various fog nodes forms the second layer of computation in our architecture. The main functionality of this layer is aggregation of tasks and collection of data from the base layer. The fog nodes disseminate the various tasks to different edge devices where the pre-processing of the huge measure of data takes place. This layer acts as a master to the base layer.

\section{Distribution of Tasks}

This assignment is executed by means of the smart gateway utilizing a job scheduler. In our implementation, circulation of various jobs is done by applying the job-scheduling algorithm for fog-cloud computational systems presented by Pham and Huh [22]. According to the algorithm, when the priority for the different tasks has been determined, these assignments are sent to nodes for execution. Presently the decision of the nodes to complete a specific task must be made figured out. For this, the time required by the processor at every node and the processing speed must be mulled over. The earliest start time and earliest finish time will be utilized for making those calculations.

\section{E. Data accumulation}

When jobs are disseminated, the data should be accumulated. Aggregation of data comprises of three fundamental divisions: mapping of the schema, data fusion, and duplicate detection. Schema mapping will guarantee that the data are accumulated in such a way that it fits with our requirements. The detection of duplicated data is a major task of these fog nodes because duplicated data leads to increase in error rates. Injection of false data is also nowadays a huge reason for reducing the efficiency. This is executed by adding a local filter to the fog device. Data fusion is the last phase of data aggregation in which the last information is accumulated and set up together as one element. As described in Fig. 4, this layer resides above the sensors layer and acts as a master to the layer beneath it. The principle objective of the fog layer is to reduce the workload of the cloud layer in order to reduce latency.

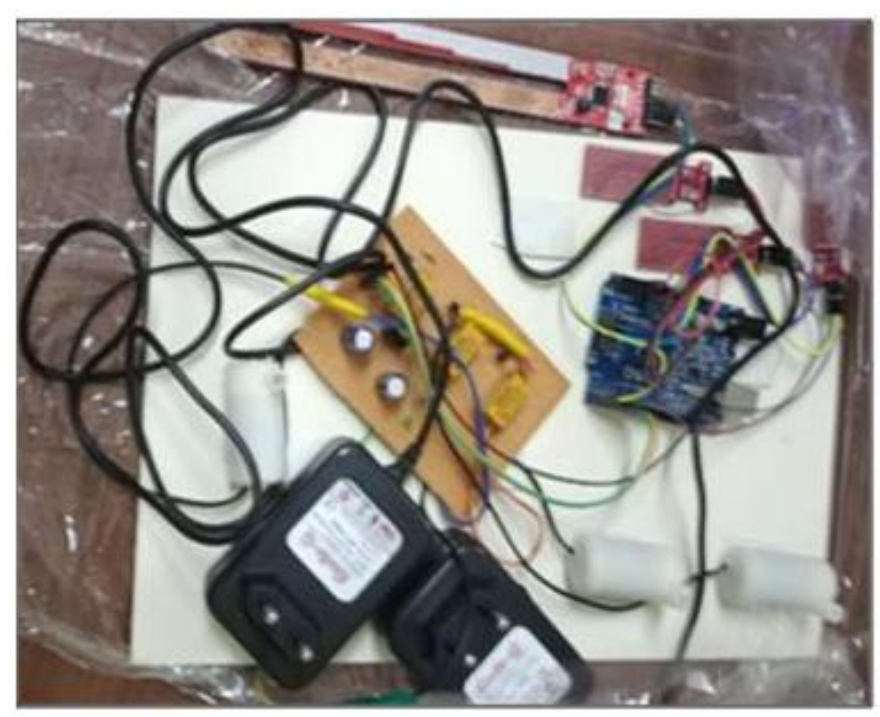

Figure 5. Node MCU connected to the sensors

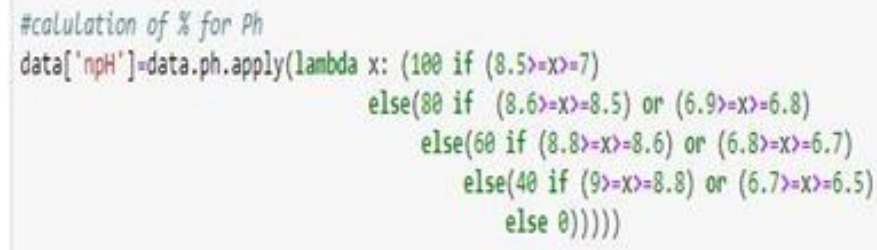

Fcalculotion of $\%$ for dissolved oxygen

data['ndo'] $=$ data.do.apply (lanbda $x:(188$ if $(x)=6)$

else $(88$ if $(6)=x)=5.1)$

else $(68$ if $(5>=x)=4.1)$

else $(48$ if $(4>-x)=3)$

else $\theta)))$ )

Figure 6. Calculation of the percentage of purity

Table 1. Drinking Water standards and unit weights. (All values except $\mathrm{pH}$ and Electrical Conductivity are in $\mathrm{mg} / \mathrm{L}$ )

\begin{tabular}{|c|c|c|c|}
\hline $\begin{array}{c}\text { Serial } \\
\text { No. }\end{array}$ & Parameters & Standards & $\begin{array}{c}\text { Unit } \\
\text { Weights }\end{array}$ \\
\hline 1. & $\mathrm{pH}$ & $6.5-8.5$ & 0.2190 \\
\hline 2. & $\begin{array}{c}\text { Electrical } \\
\text { Conductivity }\end{array}$ & 300 & 0.371 \\
\hline 3. & $\begin{array}{c}\text { Total Dissolved } \\
\text { Solids }\end{array}$ & 500 & 0.0037 \\
\hline 4. & Nitrate & 45 & 0.01236 \\
\hline 5. & Dissolved Oxygen & 5.00 & 0.3723 \\
\hline 6. & $\begin{array}{c}\text { Biological } \\
\text { Oxygen Demand }\end{array}$ & 5.00 & 0.3723 \\
\hline
\end{tabular}

According to our system, the various jobs that the fog nodes execute are described below.

\section{1) Water Quality Index (WQI) calculation:}

The quality parameters of water have different units. In order to have common line of comparison, all the parameters are converted into a single parameter alluded as Water Quality Index (WQI). The conversion takes place according to the formula. First, the percentage of purity of each parameters are calculated. This value is then used for the calculation of WQI. The overall WQI was calculated using the above equation by combining the quality rating with the unit weight linearly. Unit weight was obtained by a value inversely proportional to the recommended standard value $\mathrm{Sn}$ of the corresponding parameter.

In the above equation $\mathbf{W n}=\mathbf{K} / \mathbf{S n}$

$W Q I=\sum q n W n / \sum W n$

$\mathbf{W n}=$ unit weight for the nth parameters.

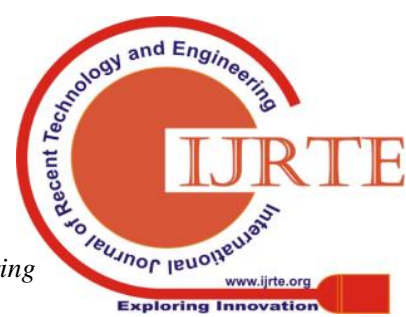


job in predicting an outcome (dependent) variable? And which variables in particular are significant predictors of the outcome variable, and in what way do they-indicated by the magnitude and sign of the beta estimates-impact the outcome variable? These regression estimates are used to explain the relationship between one dependent variable and one or more independent variables.

Table 2. Water Pricing Chart

\begin{tabular}{|c|c|c|}
\hline & $\begin{array}{l}\text { Average } \\
\text { usage of } \\
\text { water per } \\
\text { person per } \\
\text { day=0.4 kL, }\end{array}$ & $\begin{array}{l}\text { Price per } \\
\text { Kiloliter =Rs. 5 }\end{array}$ \\
\hline $\begin{array}{c}\text { Serial } \\
\text { Num ber }\end{array}$ & $\begin{array}{c}\text { Num ber of } \\
\text { Mem bers }\end{array}$ & $\begin{array}{c}\text { Stand ard free } \\
\text { limits (kL) }\end{array}$ \\
\hline \hline 1. & 1 & 0.40 \\
\hline 2. & 2 & 0.80 \\
\hline 3. & 3 & 1.20 \\
\hline 4. & 4 & 1.60 \\
\hline 5. & 5 & 2.00 \\
\hline 6. & 6 & 2.40 \\
\hline
\end{tabular}

The best fit line as depicted in Fig. 10 is found out by using the gradient descent methodology and then it is accordingly mapped. According to our data set, the regression model predicted the intercept (theta 0 ) and coefficient (theta 1) as $[74.63,2,01]$ with respect to the below given formula. $y=m x \pm c$

The above equation (2) represents the formula for finding the Best Fit Line. ' $y$ ' stands for the predicted value and ' $x$ ' stands for the actual value. The gradient is represented by ' $\mathrm{m}$ ' and $\mathrm{c}$ acts as the constant coefficient.

At the point when real-time data originates from the fog layer, it is classified under the above mentioned categories. If the quality of water is unfit for usage, the cloud performs the necessary actions (like closes the solenoid value and redirects the water to the nearest refinement plant)[23][24]. If the quality is detected as WORST, then an alert message is sent to the administration for manual checking of supply pipelines by staff.

\section{G. Water Conservation}

In order to reduce the over consumption of the water by the households, a fixed pricing chart according to the population of individual families is prepared and fed to the cloud server. The measured data received from the fog layer is compared with the pricing chart and accordingly the concerned household is charged. Also, a warning message is sent to the administration so that they can forward the information to the concerned household. This helps in preventing the over consumption of water by the family units. Fig. 11 depicts the standard water tariff that is used in these various cities of India as shown. There is no correlation to the number of members in the family which may lead to incorrect billing due to absence of standard free limit. Water consumption in a household is directly proportional to on the number of members in the household. In our system as depicted in Table 2, we have used a pricing chart which bills according to the number of members of the family.

\section{H. Security}

The security of the data is not compromised in this system because the raw data doesn't travel to and fro. The chance of tampering with the raw data gets reduced because the data gets pre-processed in the middle layers and also short term storage facility is also provided by the fog nodes.

\section{I. water conservation and quality monitoring system}

Apart from all the above mentioned layers ,our water consumption and quality monitoring system consists of the main water supply tank, the water supply pipelines, water department and the individual houses. The cloud layer after detecting the issue in the water quality sends an alert message to the concerned authority for further action with the help of the GSM module used [25][26].

\section{RESULTS AND DISCUSSION}

In the following section, the experiments performed are discussed and also analyzed with respect to the proposed system.

\section{A. Accuracy and Errors Measurement}

\begin{tabular}{|c|c|c|c|c|c|c|c|}
\hline Year & Actual WQI & Predicted WQI & Error=(Actual-Predicted)/Actual & $\operatorname{Mod}($ Error) & Percentage Error & Actual Error & SquEreror \\
\hline 2006 & 71.31 & 71.65 & 0.00 & 0.00 & 0.48 & 0.34 & 0.12 \\
\hline 2007 & 7255 & 72.43 & 0.00 & 1.00 & 99.83 & 0.12 & 0.01 \\
\hline 2008 & 72.57 & 73.20 & 0.01 & 0.01 & 0.87 & 0.63 & 0.40 \\
\hline 2009 & 74.09 & 73.98 & 0.00 & 1.00 & 99.86 & 0.10 & 0.01 \\
\hline 2010 & 74.65 & 74.76 & 0.00 & 0.00 & 0.15 & 0.11 & 0.01 \\
\hline 2011 & 75.65 & 75.54 & 0.00 & 1.00 & 99.86 & 0.11 & 0.01 \\
\hline 2012 & 78.97 & 76.32 & $\cdot 0.03$ & 0.97 & 96.64 & 265 & 7.04 \\
\hline 2013 & 75.01 & 77.09 & 0.03 & 0.03 & 278 & 208 & 4.34 \\
\hline \multirow[t]{4}{*}{2014} & 76.88 & 77.87 & 0.01 & 0.01 & 1.29 & 0.99 & 0.98 \\
\hline & & & & & 4.64 & & 1.44 \\
\hline & & & & & MAPE $=4.64$ & & MASgEETror=1.44 \\
\hline & & & & & Accuracy=55.36 & & RMSE-1.2 \\
\hline
\end{tabular}

Figure 12. Error Measurement Actual WQI vs. Predicted WQI

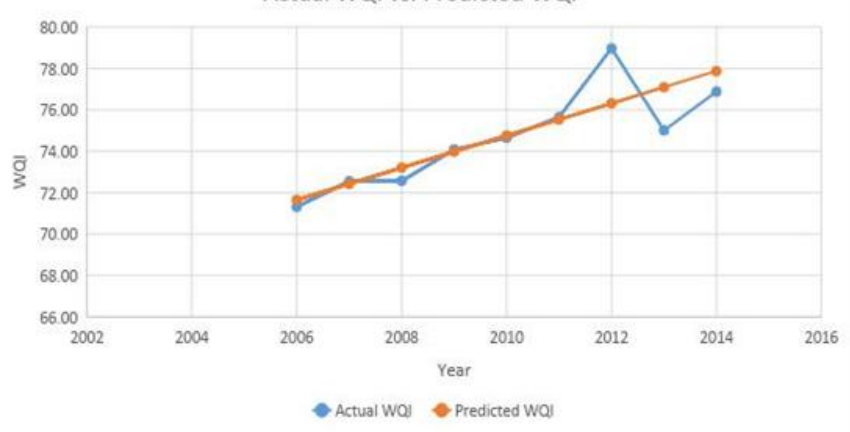

Figure 13. Actual WQI vs. Predicted WQI 
Upon completion of applying the algorithms to the data, we calculated the error values which helped us to find the accuracy also. We calculated the Mean Absolute Squared Error, Root Mean Squared Error (RMSE) and Mean Absolute Percentage Error (MAPE) as shown in Fig. 12. In Fig. 13, we have plotted the Actual WQI vs. Predicted WQI which complies with the accuracy percentile i.e. $55.36 \%$.

\section{B. Performance Analysis}

In order to compare the use of cloud computing with fog layer and without fog layer, we used iFogSim simulation toolkit to simulate the fog topology. Clouds simulation toolkit is used for the configurations which use only the cloud computing layer [27][28]. These toolkits simulated the specified topology and gave simulated results. These simulators make it very convenient for when all the necessary technological requirements are not available. Both the simulator adds a little bit of overhead time. Therefore, in order to avoid discrepancy in the results, we coordinated various tests for 5 different configurations of devices involved.

\section{Analysis of Computation}

The fog layer in our proposed system helps in the computation by pre-processing the data.The workload on the cloud layer gets reduced due to this functionality of the fog layer. The data received by the fog layer is also restrained for some time in order to avoid reloading of the same data. This reduces the network usage and the energy consumption at the datacenters, thereby results in better performance. The comparison of the traditional configuration and proposed configuration with respect to network usage is depicted in Fig. 14 below.

\section{Analysis of Latency}

The implementation of fog computing in our water conservation and quality monitoring system involves transfer of massive amount of data among the 3 tiers. In traditional systems, cloud computing alone was used to handle the data and its subsequent processing which led to increase in the latency. This heavily effected the performance. Whereas in our implementation, the presence of the fog computing layer helps in dividing the tasks between the cloud and fog layer. This in turn reduces the latency and improves performance. The comparison of the traditional configuration and proposed configuration with respect to latency is depicted in Fig.15.

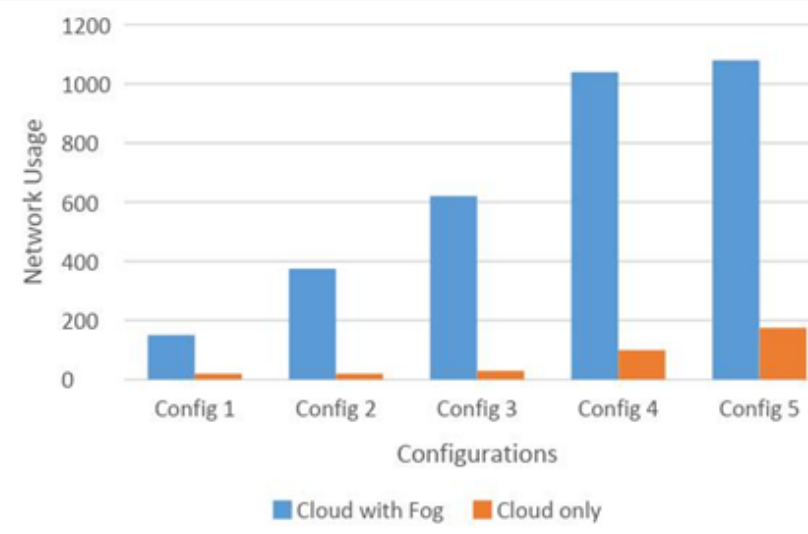

Figure 14. Network Usage Comparison

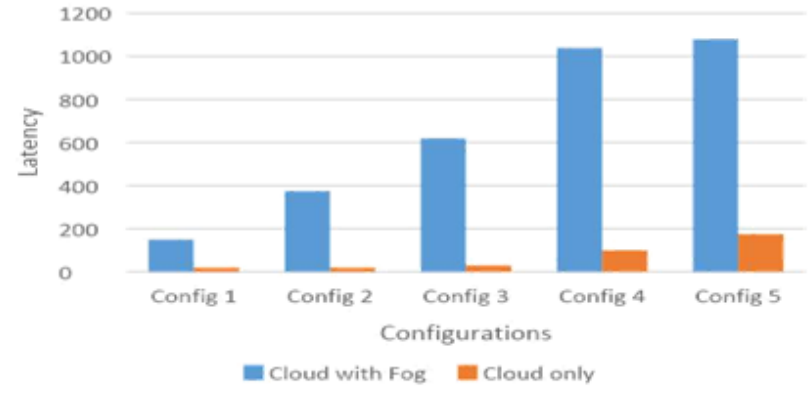

Figure 14. Latency Comparison

\section{E. Analysis of Security}

The security of the data in our system gets enhanced due to the introduction of the fog layer in the architecture. This in turn reduces the to and fro movement of the raw data between the cloud and the sensors. The raw data is pre-processed in the fog layer and then forwarded to the cloud layer for further processing and long-term storage. This reduces the probability of data tampering and data loss.

\section{CONTRIBUTION}

The effectiveness that fog computing adds to the existing water quality msonitoring and conservation systems has been highlighted in the proposed technique. Using this method, the process of preserving water and checking its quality becomes faster and more efficient. The manual labor cost is greatly reduced. This framework would be highly beneficial for areas experiencing water scarcity. Also, quality of water would also be improved for household purposes. The pricing system could be utilized to keep a check on water usage.

\section{CONCLUSION AND FUTURE WORK}

The proposed method brings to light the implementation of fog and cloud computing along with IoT to make the water quality monitoring and conservation systems much more efficient for data storage and processing. A great deal of manual labor is reduced and the systems become more reliable. For different case scenarios, different algorithms and methodologies can be used and a wide range of such applications can be readily derived. The pricing system helps in reducing over-consumption of water by households. This system can be diversified for enhanced interaction between the family units and the administration. By adding layers of fog nodes, applications can be partitioned to run at the optimal network level. This feature can be readily utilized for improving the existing water quality monitoring and conservation systems and ideating new systems in accordance with the advancement of technology.

\section{REFERENCES}

1. Bonomi F, Milito R, Natarajan P, Zhu J. 
Fog computing: A platform for internet of things and analytics. InBig data and internet of things: A roadmap for smart environments 2014 (pp. 169-186). Springer, Cham.

2. Zao JK, Gan TT, You CK, Méndez SJ, Chung CE, Te Wang Y, Mullen T, Jung TP. Augmented brain computer interaction based on fog computing and linked data. In2014 International Conference on Intelligent Environments 2014 Jun 30 (pp. 374-377). IEEE.

3. Dastjerdi AV, Buyya R. Fog computing: Helping the Internet of Things realize its potential. Computer. 2016 Aug;49(8):112-6.

4. Chen Y, Shen W, Huo H, Xu Y. A smart gateway for health care system using wireless sensor network. In2010 Fourth International Conference on Sensor Technologies and Applications 2010 Jul 18 (pp. 545-550). IEEE.

5. Hong K, Lillethun D, Ramachandran U, Ottenwälder B, Koldehofe B. Mobile fog: A programming model for large-scale applications on the internet of things. InProceedings of the second ACM SIGCOMM workshop on Mobile cloud computing 2013 Aug 16 (pp. 15-20). ACM.

6. Pham XQ, Huh EN. Towards task scheduling in a cloud-fog computing system. In2016 18th Asia-Pacific network operations and management symposium (APNOMS) 2016 Oct 5 (pp. 1-4). IEEE.

7. Gupta H, VahidDastjerdi A, Ghosh SK, Buyya R. iFogSim: A toolkit for modeling and simulation of resource management techniques in the Internet of Things, Edge and Fog computing environments. Software: Practice and Experience. 2017 Sep;47(9):1275-96.

8. Menon GS, Ramesh MV, Divya P. A low cost wireless sensor network for water quality monitoring in natural water bodies. InGlobal Humanitarian Technology Conference (GHTC), 2017 IEEE 2017 Oct 19 (pp. 1-8). IEEE.

9. Petri I, Yuce B, Kwan A, Rezgui Y. An Intelligent Analytics System for Real-Time Catchment Regulation and Water Management. IEEE Transactions on Industrial Informatics. 2018 Sep;14(9):3970-81.

10. Lambrou TP, Anastasiou CC, Panayiotou CG, Polycarpou MM. A low-cost sensor network for real-time monitoring and contamination detection in drinking water distribution systems. IEEE sensors journal. 2014 Aug;14(8):2765-72.

11. Hu C, Tian D, Yan X. Research on placement of water quality sensor in water distribution systems.
InIntelligent Control and Automation (WCICA), 2014 11th World Congress on 2014 Jun 29 (pp. 3584-3587). IEEE.

12. Aguilar JV, Langarita P, Linares L, Rodellar J. Automatic control of flows and levels in an irrigation canal. IEEE Transactions on Industry Applications. 2009 Nov;45(6):2198-208.

13. Patil K, Patil S, Patil S, Patil V. Monitoring of turbidity, pH \& temperature of water based on GSM. IJREST. 2015 Mar;2:16-21.

14. Dorini G, Jonkergouw P, Kapelan Z, Savic D. SLOTS: Effective algorithm for sensor placement in water distribution systems. Journal of Water Resources Planning and Management. 2010 Feb 26;136(6):620-8.

15. Ostfeld A, Uber JG, Salomons E, Berry JW, Hart WE, Phillips CA, Watson JP, Dorini G, Jonkergouw P, Kapelan Z, di Pierro F. The battle of the water sensor networks (BWSN): A design challenge for engineers and algorithms. Journal of Water Resources Planning and Management. 2008 Nov;134(6):556-68.

16. Hart WE, Murray R. Review of sensor placement strategies for contamination warning systems in drinking water distribution systems. Journal of Water Resources Planning and Management. 2010 Feb 19;136(6):611-9.

17. Mo D, Zhao Y, Chen S. Automatic measurement and reporting system of water quality based on GSM. InIntelligent System Design and Engineering Application (ISDEA), 2012 Second International Conference on 2012 Jan 6 (pp. 1007-1010). IEEE.

18. Kalpana MB. Online Monitoring Of Water Quality Using Raspberry Pi3 Model B. IJITR. 2016 Nov 21;4(6):4790-5.

19. Maddhesiya ${ }^{1}$ PK, Singh AP. Maintaining Drinking Water Health with Continuous Detection.

20. Computing F. the Internet of Things: Extend the Cloud to Where the Things are. Cisco White Paper. 2015.

21. Yi S, Li C, Li Q. A survey of fog computing: concepts, applications and issues. InProceedings of the 2015 workshop on mobile big data 2015 Jun 21 (pp. 37-42). ACM.

22. Teller E, Stivoric JM, Kasabach CD, Pacione CD, Moss JL, Liden CB, McCormack MA, inventors; BodyMediaInc, assignee. System for monitoring health, wellness and fitness. United States patent US 6,605,038. 2003 Aug 12. 
23. Abirami NA, Devi BM. Implementation of Sensor Nodes for Drinking Water Quality Assessment.

24. Geetha S, Gouthami S. Internet of things enabled real time water quality monitoring system. Smart Water. 2016 Dec;2(1):1.

25. Kansara K, Zaveri V, Shah S, Delwadkar S, Jani K. Sensor based automated irrigation system with IoT: a technical review. International Journal of Computer Science and Information Technologies. 2015;6(6):5331-3.

26. Mijović S, Palmar B. Continuous water quality monitoring on river Kolubara. In30th scientific professional meeting with international participation 2009.

27. Lakshmi Kanthan. N, Suresh. S, "IoT based smart water distribution management and underground pipe health monitoring system for smart city" in IEEE Conference Proceedings, IEEE sponsored 2019 5th international conference for convergence in technology, 2019.

28. Lakshmi Kanthan. N, Suresh. S, "Multi-Agent Based Water Distribution and Underground Pipe Health Monitoring System Using IoT ", Springer Nature, 16th International Conference on Information Technology- New Generations (ITNG)

\section{AUTHORS PROFILE}

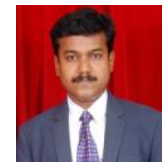

P.Selvaraj has been working as an Assistant Professor in SRMIST, Chennai for the past 13 years. He did M.Tech in IT and currently pursuing research in software defined optical networks. He published 10 papers in reputed journals. His research interest includes artificial intelligence, machine learning, pattern recognition, intelligent networks etc.,

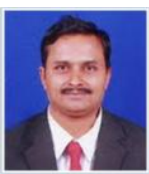

Dr. S. Prabakaran elvaraj has been working as Professor in SRMIST, Chennai for the past 12 years. He did M.E in CSE from Thapar university, Patiala and his Ph.D from IIIT, Gwalior. He published 75 papers in reputed journals. His research interest includes artificial intelligence, machine learning, pattern recognition, image processing etc.,

Sayani Chatterjee, Ambika Srivastava completed B.Tech IT from SRMIST in 2019, Chennai. They have been constantly working in the IoT based projects. 